\title{
Sur les équations fonctionnelles généralisées de Cauchy et quelques équations qui s'y rattachent
}

Par

Akira KUWAGAKI*

§. Généralités.

\subsection{Définition de l'équation fonctionnelle.}

Nous allons présenter ici quelques méthodes pour résoudre des équations fonctionnelles dérivées de l'équation de Cauchy, du point de vue générale, en réunissant nos travaux antérieurs [5-11]. Nous nous bornons ici à considérer, en général, "équation fonctionnelle" qui concient un nombre fini de relations fonctionnelles, sans opérations infinies ccmme passage aux limites ou dérivations. Donc la définition, que nous donnons ci-dessous, serait naturelle et raisonnable. L'équation aux différences finies est une équation fonctionnelle d'après notre définition, mais l'équation différentielle ou l'équation intégrale ne l'est pas [4].

Définition. Equation fonctionnelle est une égalité ou un système d'égalités formé seulement par un nombre fini de fonctions inconnues, fonctions connues et variables indépendantes. Naturellement par l'équation fonctionnelle on entend qu'elle n'est pas une identité. J. Aczél[2] et M. Kuczma [4] aussi l'ont défini de la même manière.

Exemple. Soient $x$ et $y$ des variables indépendantes, $f$ une fonction inconnue et $F$ et $G$ des fonctions données. Alors

$$
\begin{aligned}
& f(x+y)=f(x)+f(y) \quad \text { (équation de Cauchy) } \\
& f\{F(x, y)\}=G\{f(x), f(y)\}
\end{aligned}
$$

* Université Médicale de Kyoto.

Received May 23, 1966.

Communicated by K. Itô. 


$$
f\{f(x)\}=F(x)
$$

sont toutes des équations fonctionnelles.

Nous supposons en général que les variables et les fonctions sont toutes réelles (ou complexes), et que les fonctions sont continues.

Si l'on pose

$$
F(x, y)=G(x, y)=x+y
$$

dans (2), on obtient (1) de sorte que le premier exemple est un cas particulier du second. $F(x, y)$ sera appelé argument de la fonction $f(F(x, y))$.

\subsection{Classification.}

Pour examiner facilement tout le domaine de la théorie des équations fonctionnelles, nous définissons les types des équations fonctionnelles à l'aide des nombres caractéristiques qui leur appartiennent.

Rang: Nombre des variables indépendentes contenues dans une équation fonctionnelle.

Ordre: Le plus petit nombre des relations additionnelles qui sont nécessaires pour remplacer par une seule variable chacun des arguments des fonctions inconnues lesquels sont contenus dans une équation fonctionnelle. (Dans le cas de l'équation aux différences finies cette définition coïncide avec celle qui est employée ordinairement.)

Indice d'itération: Le plus grand nombre du duplicateur d'itération relative aux fonctions inconnues dans une équation fonctionnelle. (Ce nombre diffère un peu de l'indice d'implication "the implication index" de M. Kuczma [4].)

Nous appelons une équation fonctionnelle qui est du rang $r$, de l'ordre $n$ et de l'indice d'itération $i$, équation fonctionnelle du type $(r, n, i)$. Mais, ces trois nombres caractéristiques, ne suffisent pas de déterminer le nombre des constantes arbitraires ni celui des fonctions arbitraires qui sont contenues dans la solution la plus générale d'une équation fonctionnelle à laquelle ils appartiennent.

Exemple. Voici les types des équations citées au numéro 1.1:
(1)
$(2,1,1)$,
(2) $(2,1,1)$,
(3) $(1,1,2)$. 


\subsection{Généralisation de l'équation fonctionnelle de Cauchy.} L'équation fonctionrille de Cauchy

$$
f(x+y)=f(x)+f(y)
$$

est fondamentale. Nous considérons les équations fonctionnelles généralisées de Cauchy et examinons la condition pour qu'elles admettent des solutions qui ne sont pas constantes.

$1^{\circ}$ Cas où la multiplication est remplacée par l'addition.

$$
\begin{aligned}
f(x+y) & =f(x) f(y), \\
f(x y) & =f(x)+f(y), \\
f(x y) & =f(x) f(y) .
\end{aligned}
$$

Ces trois équations sont facilement résolues comme l'équation (1), et leurs solutions continues ou mesurables sont données sous les formes:

$$
\begin{array}{llrl}
f(x) & =c x, & & (c: \text { constante arbitraire }), \\
f(x) & =0, e^{c x}, & & (c: \text { constante arbitraire }), \\
f(x) & =c \log |x|, & & (c: \text { constante arbitraire) }, \\
f(x) & =0,|x|^{c},|x|^{c} \operatorname{sgn} x, & & (c: \text { constante arbitraire }) .
\end{array}
$$

$2^{\circ}$ Cas où le second membre est une fonction rationnelle de la fonction inconnue.

Si $R$ désigne une fonction rationnelle de deux variables, nous avons l'équation généralisée [5]:

$$
f(x+y)=R\{f(x), f(y)\} .
$$

Pour que cette équation admette des solutions non constantes, la fonction $R$ ne peut être arbitraire. Nous en montrerons le résultat complet au $\S 2$, où la fonction $R$ jouera un rôle de fonction inconnue.

$3^{\circ}$ Cas où la fonction inconnue est de plusieurs variables. Par exemple

$$
f\left(x_{1}+x_{2}, y_{1}+y_{2}\right)=F\left\{f\left(x_{1}, y_{1}\right), f\left(x_{1}, y_{2}\right), f\left(x_{2}, y_{1}\right), f\left(x_{2}, y_{2}\right)\right\}
$$

est une équation du type $(4,2,1)$. L'équation (8) ainsi que les équations en des variables vectorielles ou matricielles seront discutées au §3. 
$4^{\circ}$ Cas où la fonction inconnue prend la valeur vectorielle ou matricielle.

Dans ce cas, nous avons un système des équations fonctionnelles par rapport aux composantes de la fonction inconnue vectorielle ou matricielle. Il sera discuté au §3, même dans le cas où les variables et les fonctions sont toutes vecteurs ou matrices [6].

$5^{\circ}$ Cas où les variables et les fonctions sont des éléments d'une algèbre abstraite.

Récemment, l'équation multiplicative de Cauchy est considérée par W. Eichhorn [3], où les variables $x, y$ sonc des quaternions de Hamilton ou des nombres de Cayley et la fonction inconnue $f$ est un nombre réel ou un élément diune algèwre abstraite, complète et normée. C'est une généralisation d'une autre nature que la inôtre.

\section{§2. L'équation $f(x+y)=\mathbb{R}_{2}\{f(x), f(y)\}$. [5]}

\subsection{Symétrie assceiative.}

Pour que l'équation fonctionnelle

$$
f(x+y)=R\{f(x), f(y)\}
$$

admette une solution continue et non constante dans un intervalle donné, il faut et il suffit que la fonction rationnelle $R(x, y)$ soit symétrique et associative, c'est-à-dire, qu'elle doit satisfaire à deux équations fonctionnelles:

$$
\begin{array}{rlrl}
f(x, y) & =f(y, x) & & \text { (type }(2,0,1)), \\
f\{x, f(y, z)\} & =f\{f(x, y), z\} & \text { (type }(3,2,2)) .
\end{array}
$$

Nous traiterons les équations (9) et (10) au $\$ 4$.

Théorème 1. La fonction rationnelle irréductible $R(x, y)$ qui satisfait aux équations (9) et (10) se réduit à une constante où à une fonction de la forme

$$
R(x, y)=\frac{a x y+b(x+y)+c}{p x y+q(x+y)+r}
$$


où $a, b, c, p, q$ et $r$ sont des constantes telles que $a: b: c \neq p: q: r$ et

$$
\text { le rang de }\left(\begin{array}{lll}
b & a-q & p \\
c & b-r & q
\end{array}\right)=1 \text {. }
$$

DÉmonstration. Soit $m$ le degré de la fraction irréductible $R(x, y)$ en $x$. Alors le second membre de l'égalité (10) est de degré $m^{2}$ en $x$ tandis que le premier membre est de degré $m$ en $x$, d'où il résulte $m^{2}=m$. On a donc $m=0$ ou 1 .

De même, le degré de la fraction $R$ en y est 0 ou 1 . Si donc $m=1$, en vertu de la symétrie, $R(x, y)$ doit être écrit sous la forme (A), où $a: b: c \neq p: q: r$.

Si l'on pose $t=R(x, y)$ dans $R(i, z)$, on aura

$$
\begin{aligned}
& R\{R(x, y), z\} \\
= & \frac{(a z+b)\{(a x+b) y+(b x+c)\}+(b z+c)\{(p x+q) y+(q x+r)\}}{(p z+q)\{(a x+b) y+(b x+c)\}+(q z+r)\{(p x+q) y+(q x+r)\}} .
\end{aligned}
$$

Cette fraction ainsi que la fraction (A) est irréductible, et symétrique par rapport à $x, y$ et $z$. A cause de la symétrie en $x$ et $z$ des coefficients de $y$, dans le numérateur, on aura

$$
\left|\begin{array}{ll}
b & p \\
c & q
\end{array}\right|=0 \text { et }\left|\begin{array}{ll}
a-q & b \\
b-r & c
\end{array}\right|=0 .
$$

La inêrne considération relative au dénominateur nous conduit aux égalités

$$
\left|\begin{array}{cc}
a-q & p \\
b-r & q
\end{array}\right|=0 \text { et }\left|\begin{array}{ll}
p & b \\
q & c
\end{array}\right|=0 .
$$

On peut en conclure que

$$
\text { le rang de }\left(\begin{array}{lll}
b & a-q & p \\
c & b-r & q
\end{array}\right) \leqq 1 .
$$

Le rang n'étant pas égal à zéro, il est égale à 1 .

\subsection{Solution de l'équation (7).}

Théorème 2. Si l'on a (A) et (B), la relation $z=R(x, y)$ peut 
se ramener, par une transformation linéaire $t \rightarrow t^{*}$, ou bien $\grave{a} z^{*}=x^{*} y^{*}$, ou bien à $z^{*}=x^{*}+y^{*}$.

DÉmonstration. A cause des conditions relatives aux coefficients de $R(x, y)$, le système des équations algébriques en $(\xi, \eta)$ :

$$
a \xi+p \eta=\xi^{2}, \quad b \xi+q \eta=\xi \eta, \quad c \xi+r \eta=\eta^{2}
$$

ont certainement des solutions $(\xi, \eta)$ qui sont différentes de $(0,0)$.

Car, les deux premières et les deux dernières des équations (C) entraînent respectivement

$$
\left|\begin{array}{ll}
a \xi+p \eta & \xi \\
b \xi+q \eta & \eta
\end{array}\right|=0 \text { et }\left|\begin{array}{cc}
b \xi+q \eta & \xi \\
c \xi+r \eta & \eta
\end{array}\right|=0
$$

c'est-à-dire on a

$$
b \xi^{2}+(q-a) \xi \eta-p \eta^{2}=0 \text { et } c \xi^{2}+(r-b) \xi \eta-q \eta^{2}=0 .
$$

Grâce à la condition (B) ces deux èquations sont équivalentes l'une et l'autre et le système (C) a deux solutions qui scnt différentes de $(0,0)$.

On doit distinguer deux cas suivant que le système (C) admet deux solutions distinctes ou non.

$1^{\circ}$ Le cas où le système (C) a deux solutions distinctes $\left(\xi_{1}, \eta_{1}\right)$ et $\left(\xi_{2}, \eta_{2}\right)$.

On a

$$
\begin{aligned}
\frac{\xi_{1} z+\eta_{1}}{\xi_{2} z+\eta_{2}} & =\frac{\xi_{1}\{a x y+b(x+y)+c\}+\eta_{1}\{p x y+q(x+y)+r\}}{\xi_{2}\{a x y+b(x+y)+c\}+\eta_{2}\{p x y+q(x+y)+r\}} \\
& =\frac{\xi_{1}^{2} x y+\xi_{1} \eta_{1}(x+y)+\eta_{1}^{2}}{\xi_{2}^{2} x y+\xi_{2} \eta_{2}(x+y)+\eta_{2}^{2}} \\
& =\frac{\xi_{1} x+\eta_{1}}{\xi_{2} x+\eta_{2}} \cdot \frac{\xi_{1} y+\eta_{1}}{\xi_{2} y+\eta_{2}} .
\end{aligned}
$$

Cette équation se réduit à $z^{*}=x^{*} y^{*}$ par la transformation

$$
t^{*}=\frac{\xi_{1} t+\eta_{1}}{\xi_{2} t+\eta_{2}}
$$

$2^{\circ}$ Le cas où le système (C) a une solution double $(\xi, \eta)$.

Dans ce cas, nous considérons les trois courbes planes représentées 
par les équations (C). Comme ils ont une tangente commune au point $(\xi, \eta)$, le système de leurs paramètres directeurs $\left(\xi^{\prime}, \eta^{\prime}\right)$ est la solution $(\neq(0,0))$, du sysrème des équations homogène

$$
a \xi^{\prime}+p \eta^{\prime}=2 \xi \xi^{\prime}, \quad b \xi^{\prime}+q \eta^{\prime}=\xi \eta^{\prime}+\eta \xi^{\prime}, c \xi^{\prime}+r \eta^{\prime}=2 \eta \eta^{\prime} .
$$

D’après le calcul analogue à celui du cas précédent, on a

$$
\begin{aligned}
\frac{\xi^{\prime} z+\eta^{\prime}}{\xi z+\eta} & =\frac{2 \xi^{\prime} x y+\left(\xi \eta^{\prime}+\eta \xi^{\prime}\right)(x+y)+2 \eta \eta^{\prime}}{\xi^{2} x y+\xi \eta(x+y)+\eta^{2}} \\
& =\frac{(\xi y+\eta)\left(\xi^{\prime} x+\eta^{\prime}\right)+(\xi x+\eta)\left(\xi^{\prime} y+\eta^{\prime}\right)}{(\xi x+\eta)(\xi y+\eta)} \\
& =\frac{\xi^{\prime} x+\eta^{\prime}}{\xi x+\eta}+\frac{\xi^{\prime} y+\eta^{\prime}}{\xi y+\eta}
\end{aligned}
$$

et par la transformation linéaire

$$
t^{*}=\frac{\xi^{\prime} t+\eta^{\prime}}{\xi t+\eta}
$$

l'équation $z=R(x, y)$ se réduit à $z^{*}=x^{*}+y^{*}$.

C.Q.F.D.

La transformation linéaire $t=f(s) \rightarrow t^{*}=f^{*}(s)$ amène l'équation (7) à (4) ou à (1), c'est-à-dire, on a

$$
f^{*}(x+y)=f^{*}(x) f^{*}(y) \text { ou } f^{*}(x+y)=f^{*}(x)+f^{*}(y)
$$

et leurs soluitions continues (ou mesurables) sont

$$
f^{*}(x)=e^{c_{1} x} \quad \text { ou } \quad f^{*}(x)=c_{1} x
$$

respectivement, où $c_{1}$ est une constante arbitraire.

Nous pouvons donc énoncer le théorème principal suivant.

Théorème 3. Pour que l'équation fonctionnelle (7) admette une solution continue: (ou mesurable) non constante, il faut et il suffit que la fonction $R$ soit de la forme (A) du théorème 1.

De plus, la solution de (7) s'écrit sous la forme

$$
f(x)=\frac{\eta_{2} e^{c_{1} x}-\eta_{1}}{-\tilde{s}_{2} e^{c_{1} x}+\xi_{1}} \quad \text { ou } \quad f(x)=\frac{c_{1} \eta x-\eta^{\prime}}{-c_{1} \xi x+\xi^{\prime}}
$$

suivant que le système des équations (C) admet deux racines distincte; 
$\left(\xi_{1}, \eta_{1}\right),\left(\xi_{2}, \eta_{2}\right)$ ou une racine double $(\xi, \eta)$, où $c_{1}$ est une constante arbitraire et $\left(\xi^{\prime}, \eta^{\prime}\right)$ satisfait au système des équations $\left(\mathrm{C}^{\prime}\right)$.

\subsection{Généralisation de l'équation (7).}

Soient $R_{1}(x, y)$ et $R_{2}(x, y)$ des fonctions rationnelles, symétriques et associatives, et considérons l'équation

$$
\left.f\left\{R_{1}(x, y)\right\}=R_{2}\{f(x), f(y)\} \quad \text { (type }(2,1,1)\right)
$$

qui est plus générale que (7).

D'après le théorème 2 , la relation $z=R_{1}(x, y)$ se ramène, par une certaine transformation linéaire $t \rightarrow t^{*}$, ou bien à $z^{*}=x^{*} y^{*}$ ou bien à $z^{*}=x^{*}+y^{*}$. La relation (11) se transforme alors à

ou à

$$
f^{*}\left(x^{*} y^{*}\right)=R_{2}\left\{f^{*}\left(x^{*}\right), f^{*}\left(y^{*}\right)\right\},
$$

$$
f^{*}\left(x^{*}+y^{*}\right)=R_{2}\left\{f^{*}\left(x^{*}\right), f^{*}\left(y^{*}\right)\right\} .
$$

En appliquant de nouveau le théorème 2, l'équation (11) se réduit à une des quatre équations (1), (4), (5) et (6). Donc, l'équation (11) peut être résolue à l'aide du théorème 3 .

\section{§3. Fonctions inconnues de plusieurs variables.}

\subsection{Cas de deux variables. [7]}

Dans le cas où la fonction $F$ de l'équation fonctionnelle (8) est une fonction rationnelle, nous avons l'équation

$$
f\left(x_{1}+x_{2}, y_{1}+y_{2}\right)=R\left\{f\left(x_{1}, y_{1}\right), f\left(x_{1}, y_{2}\right), f\left(x_{2}, y_{1}\right), f\left(x_{2}, y_{2}\right)\right\} \text {. }
$$

Cette équation admet une solution non constante, pour la fraction $R$ du degré arbitraire. Relativement au degré de $\mathrm{R}$, il n'est nécessaire de poser aucune restriction qui était nécessaire dans le cas d'une variable, comme on le voit sur les exemples suivants.

Exemple 1. L'équation

$$
\begin{aligned}
& f\left(x_{1}+x_{2}, y_{1}+y_{2}\right) \\
= & \frac{1}{2}\left\{f\left(x_{1}, y_{1}\right)+f\left(x_{1}, y_{2}\right)+f\left(x_{2}, y_{1}\right)+f\left(x_{2}, y_{2}\right)\right\} \\
& +\left\{f\left(x_{1}, y_{1}\right)-f\left(x_{1}, y_{2}\right)-f\left(x_{2}, y_{1}\right)+f\left(x_{2}, y_{2}\right)\right\} R\{\cdots\}
\end{aligned}
$$


admet la solution

$$
f(x, y)=c_{1} x+c_{2} y, \quad\left(c_{1}, c_{2}: \text { constantes arbitraires }\right)
$$

quelle que soit la fonction

$$
R\{\cdots\}=R\left\{f\left(x_{1}, y_{1}\right), f\left(x_{1}, y_{2}\right), f\left(x_{2}, y_{1}\right), f\left(x_{2}, y_{2}\right)\right\} .
$$

Exemple 2. L'équation

$$
\begin{aligned}
& f\left(x_{1}+x_{2}, y_{1}+y_{2}\right) \\
= & f\left(x_{1}, y_{1}\right)+f\left(x_{1}, y_{2}\right)+f\left(x_{2}, y_{1}\right)+f\left(x_{2}, y_{2}\right) \\
& +\left\{f\left(x_{1}, y_{1}\right) f\left(x_{2}, y_{2}\right)-f\left(x_{1}, y_{2}\right) f\left(x_{2}, y_{1}\right)\right\} R\{\cdots\}
\end{aligned}
$$

admet la solution

$$
f(x, y)=c x y, \quad(c: \text { constante arbitraire })
$$

quelle que soit la fonction $R\{\cdots\}$.

Exemple 3. L'équation

$$
f\left(x_{1}+x_{2}, y_{1}+y_{2}\right)=f\left(x_{1}, y_{1}\right) f\left(x_{1}, y_{2}\right) f\left(x_{2}, y_{1}\right) f\left(x_{1}, y_{2}\right)
$$

admet la solution

$$
f(x, y)=k e^{c x y}, \quad\left(c: \text { constante arbitraire, } k^{4}=k\right) .
$$

Cela posé, nous dirons que nous pouvons résoudre complètement l'équation (12) pourvu que chacune des variables $x_{1}, x_{2}, y_{1}$ et $y_{2}$ se trouve symboliquement au plus une fois dans chaque terme du numérateur et du dénominateur de $R$. Dans ce cas, nous débarrassons le dénominateur en le multipliant aux deux membres de l'équation (12) et permutons $x_{1}$ et $x_{2}$ ainsi que $y_{1}$ et $y_{2}$. Puis ajoutons les quatre égalités ainsi obtenues et divisons la somme par quatre. Nous aurons, sans perdre la généralité, l'équation fonctionnelle symétrique suivante

$$
\begin{aligned}
& f\left(x_{1}+x_{2}, y_{1}+y_{2}\right) \\
& a\left\{f\left(x_{1}, y_{1}\right) f\left(x_{2} y_{2}\right)+f\left(x_{1}, y_{2}\right) f\left(x_{2}, y_{1}\right)\right\} \\
&= \frac{+b\left\{f\left(x_{1}, y_{1}\right)+f\left(x_{1}, y_{2}\right)+f\left(x_{2}, y_{1}\right)+f\left(x_{2}, y_{2}\right)\right\}+2 c}{p\left\{f\left(x_{1}, y_{1}\right) f\left(x_{2}, y_{2}\right)+f\left(x_{1}, y_{2}\right) f\left(x_{2}, y_{1}\right)\right\}} \\
&+q\left\{f\left(x_{1}, y_{1}\right)+f\left(x_{1}, y_{2}\right)+f\left(x_{2}, y_{1}\right)+f\left(x_{2}, y_{2}\right)\right\}+2 r
\end{aligned}
$$


où $a, b, c, p, q$ et $r$ sont des constantes et $a: b: c \neq p: q: r$.

Théorème 4. Pour que l'équation fonctionnelle (16) admetie une solution $f(x, y)$ uniforme et continue dans un voisinage du point $(0,0)$

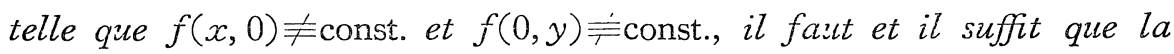
condition (B) du théorème 1 soit remplie.

Théorème 5. Sous la condition (B), les solutions de l'équation (16) doivent être égales aux fractions linéaires de $c_{1} x+c_{2} y$ ou de $e^{c_{1} x+c_{2} y}, c_{1}$ et $c_{2}$ étant deux constantes arbitraires.

On peut démontrer ces deux théorèrnes tout ensemble, et on peut trouver toutes les solutions de (16) par les procédés suivants.

Démonstrations. Posons d'abord $y_{1}=y_{2}=0$ dans l'équation (16). Nous avons l'équation fonctionnelle en $f(x, 0)$ :

$$
f\left(x_{1}+x_{2}, 0\right)=\frac{a f\left(x_{1}, 0\right) f\left(x_{2}, 0\right)+b\left\{f\left(x_{1}, 0\right)+\hat{J}\left(x_{2}, 0\right)\right\}+c}{p f\left(x_{1}, 0\right) f\left(x_{2}, 0\right)+q\left\{f\left(x_{1}, 0\right)+f\left(x_{2}, 0\right)\right\}+r} .
$$

Puisqu'on suppose $f(x, 0) \stackrel{\prime}{\neq}$ const., le théorème 3 montre la nécessité de la condition (B).

Puis, si la condition (B) est remplie, le système des équations (C) en $(\xi, \eta)$ admet des solutions différentes de $(0,0)$. Deux cas sont à distinguer suivant que le système admet deux solutions distinctes ou une solution double.

$1^{\circ}$ Cas où le système (C) a deux solutions distinctes $\left(\xi_{1}, \eta_{1}\right)$ et $\left(\xi_{2}, \eta_{2}\right)$.

Les relations (16) et (C) entraînent

$$
\frac{\xi_{1} f\left(x_{1}+x_{2}, y_{1}+y_{2}\right)+\eta_{1}}{\xi_{2} f\left(x_{1}+x_{2}, y_{1}+y_{2}\right)+\eta_{2}}
$$

$$
=\frac{\left\{\xi_{1} f\left(x_{1}, y_{1}\right)+\eta_{1}\right\}\left\{\xi_{1} f\left(x_{2}, y_{2}\right)+\eta_{1}\right\}+\left\{\xi_{1} f\left(x_{1}, y_{2}\right)+\eta_{1}\right\}\left\{\xi_{1} f\left(x_{2}, y_{1}\right)+\eta_{1}\right\}}{\left\{\xi_{2} f\left(x_{1}, y_{1}\right)+\eta_{2}\right\}\left\{\xi_{2} f\left(x_{2}, y_{2}\right)+\eta_{2}\right\}+\left\{\xi_{2} f\left(x_{1}, y_{2}\right)+\eta_{2}\right\}\left\{\xi_{2} f\left(x_{2}, y_{1}\right)+\eta_{2}\right\}} .
$$

En y posant $y_{1}=y_{2}=0$, on obtient

$$
\frac{\xi_{1} f\left(x_{1}+x_{2}, 0\right)+\eta_{1}}{\xi_{2} f\left(x_{1}+x_{2}, 0\right)+\eta_{2}}=\frac{\xi_{1} f\left(x_{1}, 0\right)+\eta_{1}}{\xi_{2} f\left(x_{1}, 0\right)+\eta_{2}} \cdot \frac{\tilde{\xi}_{1} f\left(x_{2}, 0\right)+\eta_{1}}{\xi_{2} f\left(x_{2}, 0\right)+\eta_{2}}
$$


et la solution est donnée par

$$
\frac{\xi_{1} f(x, 0)+\eta_{1}}{\xi_{2} f(x, 0)+\eta_{2}}=e^{c_{1} x}, \quad\left(c_{1}: \text { constante arbitraire }\right) .
$$

De même, en permutant les rôles de $x$ et $y$, on obtient.

$$
\frac{\xi_{1} f(0, y)+\eta_{1}}{\xi_{2} f(0, y)+\eta_{2}}=e^{c_{2} y}, \quad\left(c_{2}: \text { constante arbitraire }\right) .
$$

On a ensuite

$$
\frac{\xi_{1} f(0,0)+\eta_{1}}{\xi_{2} f(0,0)+\eta_{2}}=1
$$

Alors, posons $x_{2}=y_{2}=0$ dans l'équation $\left(16^{*}\right)$. A l'aide de ces trois relations ainsi obtenues, on obtient

$$
\begin{aligned}
& \frac{\xi_{1} f(x, y)+\eta_{1}}{\xi_{2} f(x, y)+\eta_{2}} \\
& =\frac{\left\{\xi_{1} f(x, y)+\eta_{1}\right\}\left\{\xi_{1} f(0,0)+\eta_{1}\right\}+\left\{\xi_{1} f(x, 0)+\eta_{1}\right\}\left\{\xi_{1} f(0, y)+\eta_{1}\right\}}{\left\{\xi_{2} f(x, y)+\eta_{2}\right\}\left\{\xi_{2} f(0,0)+\eta_{2}\right\}+\left\{\xi_{2} f(x, 0)+\eta_{2}\right\}\left\{\xi_{2} f(0, y)+\eta_{2}\right\}} \\
& =\frac{\xi_{1} f(x, 0)+\eta_{1}}{\xi_{2} f(x, 0)+\eta_{2}} \cdot \frac{\xi_{1} f(0, y)+\eta_{1}}{\xi_{2} f(0, y)+\eta_{2}}=e^{c_{1} x} \cdot e^{c_{2} y}=e^{c_{1} x^{ \pm} c_{2} y}
\end{aligned}
$$

Donc, il en résulte que

$$
f(x, y)=\frac{\eta_{2} e^{c_{1} x+c_{2} y}-\eta_{1}}{-\xi_{2} e^{c_{1} x+c_{2} y}-\xi_{1}}
$$

La fonction $\left(16^{\prime}\right)$ satisfait sûrement à l'équation (16).

$2^{\circ}$ Cas où le système $(\mathrm{C})$ a une solution double $(\xi, \eta)$.

Dans ce cas le système des équations homogènes $\left(\mathrm{C}^{\prime}\right)$ en $\left(\xi^{\prime}, \eta^{\prime}\right)$ admet une solution $\left(\xi^{\prime}, \eta^{\prime}\right)$ différente de $(0,0)$.

D'après les relations (16), (C) et $\left(\mathrm{C}^{\prime}\right)$, on a

$$
\begin{aligned}
\left(16^{* *}\right) & \frac{\xi^{\prime} f\left(x_{1}+x_{2}, y_{1}+y_{2}\right)+\eta^{\prime}}{\xi f\left(x_{1}+x_{2}, y_{1}+y_{2}\right)+\eta} \\
& \left\{\xi f\left(x_{1}, y_{1}\right)+\eta\right\}\left\{\xi^{\prime} f\left(x_{2}, y_{2}\right)+\eta^{\prime}\right\}+\left\{\xi^{\prime} f\left(x_{1}, y_{1}\right)+\eta^{\prime}\right\}\left\{\xi f\left(x_{2}, y_{2}\right)+\eta\right\} \\
= & \frac{+\left\{\xi f\left(x_{1}, y_{2}\right)+\eta\right\}\left\{\xi^{\prime} f\left(x_{2}, y_{1}\right)+\eta^{\prime}\right\}+\left\{\xi^{\prime} f\left(x_{1}, y_{2}\right)+\eta^{\prime}\right\}\left\{\xi f\left(x_{2}, y_{1}\right)+\eta\right\}}{\left\{\xi f\left(x_{1}, y_{1}\right)+\eta\right\}\left\{\xi f\left(x_{2}, y_{2}\right)+\eta\right\}+\left\{\xi f\left(x_{1}, y_{2}\right)+\eta\right\}\left\{\xi f\left(x_{2}, y_{1}\right)+\eta\right\}}
\end{aligned}
$$


Celle-ci se réduit à l'équation de Cauchy

$$
\begin{aligned}
& \frac{\xi^{\prime} f\left(x_{1}+x_{2}, 0\right)+\eta^{\prime}}{\xi f\left(x_{1}+x_{2}, 0\right)+\eta} \\
& \quad=\frac{\xi^{\prime} f\left(x_{1}, 0\right)+\eta^{\prime}}{\xi f\left(x_{1}, 0\right)+\eta}+\frac{\xi^{\prime} f\left(x_{2}, 0\right)+\eta^{\prime}}{\xi f\left(x_{2}, 0\right)+\eta},
\end{aligned}
$$

si l'on y pose $y_{1}=y_{2}=0$. La solution est alors donnée par

$$
\frac{\xi^{\prime} f(x, 0)+\eta^{\prime}}{\xi f(x, 0)+\eta}=c_{1} x, \quad\left(c_{1} \text { : constante arbitraire }\right) \text {. }
$$

On a de même

$$
\begin{aligned}
\frac{\xi^{\prime} f(0, y)+\eta^{\prime}}{\xi f(0, y)+\eta}=c_{2} y \quad \text { ou } \quad & \frac{\xi^{\prime} f(0,0)+\eta^{\prime}}{\xi f(0,0)+\eta}=0, \\
& \left(c_{2}: \text { constante arbitraire }\right),
\end{aligned}
$$

en posant $x_{1}=x_{2}=0$ ou $x_{1}=x_{2}=y_{1}=y_{2}=0$ respectivement.

Puis, en posant $x_{2}=y_{2}=0$ dans l'équation (16**) et en tenant compte de ces trois relations, on obtient

$$
\frac{\xi^{\prime} f(x, y)+\eta^{\prime}}{\xi f(x, y)+\eta}=\frac{\xi^{\prime} f(x, 0)+\eta^{\prime}}{\xi f(x, 0)+\eta}+\frac{\xi^{\prime} f(0, y)+\eta^{\prime}}{\xi f(0, y)+\eta}=c_{1} x+c_{2} y .
$$

Donc, on a enfin

$$
f(x, y)=\frac{\eta\left(c_{1} x+c_{2} y\right)-\eta^{\prime}}{-\xi\left(c_{1} x+c_{2} y\right)+\xi^{\prime}}
$$

La fonction $\left(16^{\prime \prime}\right)$ aussi satisfait sûrement à l'équation (16). Puisque les relations $\left(16^{\prime}\right)$ et $\left(16^{\prime \prime}\right)$ donnent les solutions les plus générales dans les cas respectifs, on a établi le théorème 5 .

\subsection{Formule d'addition algébrique.}

Comme l'éxtension de la formule d'addition algébrique pour une fonction d'une variable:

$$
P\{f(x+y), f(x), f(y)\}=0,
$$

où $P$ désigne un polynome, nous considérons le cas de deux variables [8].

$$
P\left\{f\left(x_{1}+x_{2}, y_{1}+y_{2}\right), f\left(x_{1}, y_{1}\right), f\left(x_{1}, y_{2}\right), f\left(x_{2}, y_{1}\right), f\left(x_{2}, y_{2}\right)\right\}=0
$$


Nous donnons une méthode pour résoudre l'équation (18) en supposant l'existence de la solution de (17).

La solution de (18) peut s'obtenir par les procédés suivants.

$1^{\circ}$ En posant $x_{1}=x_{2}=y_{1}=y_{2}=0$, on obtient une équation algébrique en

$$
k=f(0,0) .
$$

On prend pour $k$ une de ses racines.

$2^{\circ}$ En posant $y_{1}=y_{2}=0$, on obtient une formule d'addition relative à la fonction d'une seule variable $x$ :

$$
F(x)=f(x, 0) .
$$

On prend pour $F$ une de ses solutions.

$3^{\circ}$ En posant $x_{1}=x_{2}=0$, on obtient une formule d'addition relative à la fonction d'une seule variable $y$ :

$$
G(y)=f(0, y) .
$$

On prend pour $G$ une de ses solutions.

$4^{\circ}$ Enfin, posons $x_{2}=y_{2}=0$. On a la relation

$$
P\{f(x, y), f(x, y), F(x), G(y), k\}=0 .
$$

Si $f(x, y)$ ne disparaît pas dans cette équation, on résout algébriquement cette équation par rapport à $f(x, y)$.

$5^{\circ}$ On cherche enfin la condition pour que la fonction ainsi obtenue satisfasse à l'éqation donnée (18).

\section{3. Cas où les variables sont des vecteurs ou des matrices.}

L'équation fonctionnelle généralisée de (7):

$$
f(x+y)=R\{f(x), f(y)\},
$$

où $x$ et $y$ sont des vecteurs ou des matrices, peut être résolue par le même procédé que dans le cas où les variables sont des nombres. L'équation $\left(7^{*}\right)$ se réduit, par une transformation linéaire, à

$$
f^{*}(x+y)=f^{*}(x) f^{*}(y),
$$

ou à 


$$
f^{*}(x+y)=f^{*}(x)+f^{*}(y) .
$$

Les solutions de (19) ou de (20) sont

$$
\begin{aligned}
f^{*}(x)= & e^{c x}=e^{c_{1} x_{1}+c_{2} x_{2}+\cdots+c_{m} x_{m}}, \\
& \left(c_{1}, c_{2}, \cdots, c_{m}: \text { constantes arbitraires }\right),
\end{aligned}
$$

ou

$$
\begin{aligned}
f^{*}(x)= & c x=c_{1} x_{1}+c_{2} x_{2}+\cdots+c_{m} x_{m}, \\
& \left(c_{1}, c_{2}, \cdots, c_{m}: \text { constantes arbitraires }\right),
\end{aligned}
$$

respectivement. Par conséquent, toutes les solutions de l'équation $\left(7^{*}\right)$ sont obtenues. Dans le cas de $m \times n$-matrices, on pose

$$
c x=c_{11} x_{11}+c_{12} x_{12}+\cdots+c_{m n} x_{m n},
$$

au lieu de

$$
c x=c_{1} x_{1}+c_{2} x_{2}+\cdots+c_{m} x_{m},
$$

du cas de vecteur.

\subsection{Cas oin les fonctions inconnues sont des vecteurs ou des matrices carrées.}

Puisque l'on n'a pas pour le produit de deux vecteurs à dimension quelconque un vecteur ayant la même dimension, l'extension possible de l'équation de Cauchy est seulement l'équation

$$
f(x+y)=f(x)+f(y),
$$

dont la solution s'écrit sous la forme

$$
\begin{aligned}
f(x)= & c_{1} x_{1}+c_{2} x_{2}+\cdots+c_{m} x_{m}, \\
& \left(c_{1}, c_{2}, \cdots, c_{m}: \text { vecteurs constants }\right) .
\end{aligned}
$$

Scient $X$ et $Y$ des variables de $m \times m$-matrices et $F$ une fonction inconnue de $n \times n$-matrice, les quatre équations fonctionnelles:

$$
\begin{aligned}
F(X+Y) & =F(X)+F(Y), \\
F(X+Y) & =F(X) F(Y), \\
F(X Y) & =F(X)+F(Y), \\
F(X Y) & =F(X) F(Y),
\end{aligned}
$$


ont été étudiées dant notre travail [6], où nous avons donné les solutions complètes. Parmi elles la dernière est la plus essentielle. Elle est recherchée aussi par $\mathrm{O}$. Perron [12]. La première est évidemment équivalente à (21).

\section{$\$ 4 . \quad$ Symétrie et as'sociativité.}

\section{1. Symétrie.}

Considérons l'équation fonctionnelle de la symétrie

$$
f(x, y)=f(y, x) .
$$

Sa solution rationnelle est une fonction rationnelle avec les coefficients symétriques en $x$ et $y$. Dans le cas général, il est clair que la solution générale est

$$
f(x, y)= \begin{cases}f_{0}(y, x), & (x>y), \\ f_{0}(x, y), & (x \leqq y) .\end{cases}
$$

où $f_{0}(x, y)$ est une fonction arbitraire donnée dans le domaine $x \leqq y$.

\section{2. Associativité. [9]}

Le but principal de $\$ 4$ est d'étudier l'équation de l'associativité

$$
f\{x, f(y, z)\}=f\{f(x, y), z\} .
$$

C'est un exemple de l'équation à l'indice d'itération 2.

Nous allons maintenant étudier la fonction analytique de deux variables complexes: $f(x, y)$ satisfaisant à l'équation (10). Nous supposerons qu'il y ait au moins un nombre complexe $c$, fini ou infini, tel que

$$
f(c, c)=c,
$$

et que $f(x, y)$ ait un développement de Taylor à $(c, c)$. Mais, le nombre $c$ peut être réduit facilement à 0 par le moyen suivant, sans altérer les relations (10) et (D).

$1^{\circ}$ Si $c$ est un nombre fini, posons

$$
f_{1}(x, y) \equiv f(x+c, y+c)-c .
$$

Nous avons par (10) 
et $\operatorname{par}(\mathrm{D})$

$$
\begin{aligned}
f_{1}\left\{x, f_{1}(y, z)\right\} & =f_{1}\{x, f(y+c, z+c)-c\} \\
& =f\{x+c, f(y+c, z+c)\}-c \\
& =f\{f(x+c, y+c), z+c\}-c \\
& =f_{1}\{f(x+c, y+c)-c, z\} \\
& =f_{1}\left\{f_{1}(x, y), z\right\},
\end{aligned}
$$

$$
f_{1}(0,0)=f(c, c)-c=0 .
$$

$2^{\circ}$ Si $c$ est infini, posons

$$
f_{2}(x, y) \equiv \frac{1}{f(1 / x, 1 / y)} .
$$

Nous avons par (10)

$$
\begin{aligned}
f_{2}\left\{x, f_{2}(y, z)\right\} & =\frac{1}{f\{1 / x, f(1 / y, 1 / z)\}} \\
& =\frac{1}{f\{f(1 / x, 1 / y), 1 / z\}} \\
& =f_{2}\left\{f_{2}(x, y), z\right\}
\end{aligned}
$$

et par (D)

$$
f_{2}(0,0)=\frac{1}{f(\infty, \infty)}=0 .
$$

\section{3. Théorèmes principanx.}

Nous énonçons un théorème principal sous la condition que l'on ait $\left(D^{*}\right)$

$$
f(0,0)=0 \text {, }
$$

au lieu de (D).

Théorème 6. Si la fonction de deux variables complexes $f(x, y)$ est holomorphe à $(0,0)$ et satisfait à l'équation (10) et à la condition $\left(\mathrm{D}^{*}\right)$, elle appartient à une des quatre espèces des fonctions suivantes:

$$
f(x, y) \equiv x,
$$

3)

$f(x, y) \equiv y$,

4)

$$
f(x, y) \equiv x+y+x y S_{1}(x, y) \text {, }
$$

$$
f(x, y) \equiv x y S_{2}(x, y) \text {, }
$$


où $S_{1}$ et $S_{2}$ sont holomorphes à $(0,0)$, symétriques par rapport à $x$ et $y$, et $S_{2}(0,0) \neq 0$ ou $S_{2}(x, y) \equiv 0$.

J. Aczél [1] a démontré un théorème sur la fonction de deux variables réelles dont la valeur est aussi comprise dans $(a, b)$ pour chaque $x$ et $y$ de l'intervalle réel $(a, b)$.

Théorème (Aczél). Pour qu'il existe une fonction $\varphi(z)$ croissante, continue, définie sur l'intervalle réel $(a, b)$ et permettant de mettre $f(x, y)$ sous la forme

$$
f(x, y)=\varphi^{-1}\{\varphi(x)+\varphi(y)\},
$$

il faut et il suffit que la fonction $f$ soit continue, associative et monotonement croissante en $x$ et en $y$.

Dans ce théorème, l'opération $f(x, y)$ définit un groupe continu, grâce à la monotonité et la continuité de $\varphi$. Dans notre théorème 6 l'opération $f(x, y)$ définit un groupe seulement si l'on a 3 ) ; si au contraire on a 4) elle ne définit qu'un semi-groupe, parce qu'il n'y a pas d'opération inverse. De plus, on pourra obtenir sans peine directement le résultat suivant, à l'aide du théorème 6 .

Théorème 7. Un semi-groupe de dimension un, analytique au point $c$ et tel que le produit de c par luì-même est égal à c lui-même, est commutatif (c'est-à-dire abélien).

\section{4. Démonstration du théorème 6 .}

$1^{\circ}$ La fonction $f(x, y)$ étant holomorphe, on a le développement $\operatorname{par}\left(D^{*}\right)$

$$
f(x, y)=\sum_{i=1}^{\infty} H_{i}(x, y)
$$

où $H_{i}(x, y)$ est un polynome homogène du degré $i$ de $x$ et $y$. En posant $y=z=0$ dans l'équation (10), on obtient

$$
f(x, 0)=f\{f(x, 0), 0\} .
$$

Puis, développons les deux membres de (E) à l'aide du développement: 


$$
f(x, 0)=\sum_{i=\mu}^{\infty} h_{i} x^{i}
$$

et on peut supposer $h_{\mu} \neq 0$ sauf le cas de $f(x, 0) \equiv 0$. On a, en comparant les termes du moindre degré en $x$,

$$
h_{\mu} x^{\mu}=h_{\mu}^{1+\mu} x^{\mu^{2}}
$$

d'où $\mu^{2}=\mu$, et puisqu'on suppose $\mu>0$, on a $\mu=1$, et puis

$$
h_{\mu}=h_{\mu}^{2} \text {. }
$$

On a par suite $h_{1}=1$, et ensuite

$$
f(x, 0) \equiv x+\sum_{i=\nu}^{\infty} h_{i} x^{i}, \quad(\nu \geqq 2) .
$$

En portant cette relation dans (E) on obtient

$$
f(x, 0) \equiv f(x, 0)+\sum_{i=\nu}^{\infty} h_{i}\left(x+\sum_{j=\nu}^{\infty} h_{j} x^{j}\right)^{i}
$$

ou

$$
\sum_{i=\nu}^{\infty} h_{i}\left(x+\sum_{j=\nu}^{\infty} h_{j} x^{j}\right) \equiv 0, \quad(\nu \geqq 2) .
$$

Le coefficient du terme du degré $\nu$, étant $h_{\nu}$, on a $h_{\nu}=0$. On en conclut $f(x, 0) \equiv x$.

Par conséquent, on a

$$
f(x, 0) \equiv 0 \quad \text { ou } \quad x .
$$

On a de même,

$$
f(0, y) \equiv 0 \quad \text { ou } \quad y .
$$

On en conclut que les quatre cas suivants sont possibles;
a)
$f(x, y) \equiv x+\sum_{i=2}^{\infty} H_{i}(x, y)$,
b)
$f(x, y) \equiv y+\sum_{i=2}^{\infty} H_{i}(x, y)$,
c)
$f(x, y) \equiv x+y+x y \sum_{i=0}^{\infty} H_{i}(x, y)$,
d)

$$
f(x, y) \equiv x y \sum_{i=0}^{\infty} H_{i}(x, y),
$$


$H_{i}\left(x, y^{\prime}\right)$ étant un polynome homogène du degré $i$ en $x$ et $y$.

$2^{\circ}$ Le cas a). Supposons l'existence d'un nombre naturel $i$ tei que

$$
H_{2} \equiv H_{3} \equiv \cdots \equiv H_{n-1} \equiv 0 \text { et } H_{n} \doteq 0, \quad(n \geqq 2) .
$$

On a, par la relation (10),

$$
\begin{aligned}
& x+\sum_{i=n}^{\infty} H_{i}\left\{x, y+\sum_{j=1}^{\infty} H_{j}(y, z)\right\} \\
= & x+\sum_{i=n}^{\infty} H_{i}(x, y)+\sum_{i=n}^{\infty} H_{i}\left\{x+\sum_{j=, l}^{\infty} H_{j}(x, y), z\right\} .
\end{aligned}
$$

Par la comparaison des termes du degré $n$ des deux membres, on aurait

$$
H_{n}(x, y) \equiv H_{n}(x, y)+H_{n}(x, z),
$$

c'est-à-dire,

$$
H_{n}(x, z) \equiv 0,
$$

contrairement à notre hypothèse.

Donc, nous obtenons 1) du théorème 6. Dans le cas b), nous obtenons de même 2) du théorème 6 .

$3^{\circ}$ Le cas c). En différentiant par rapport à $x$ l'équation c), nous avons $f_{x}(0,0)=1 \div 0$. Par suite, l'équation en $x: z=f(x, y)$ a une solution unique $x=\varphi(y, z)$ dans un voisinage de $(0,0)$. L'équation c) est donc une représentation d'un groupe de Lie de dimension un, dont le produit est la fonction $f(x, y)$ et l'unité 0 . On en conclut facilement la symétrie $f(x, y)=f(y, x)$ et 3$)$ du théorème 6 .

$4^{\circ}$ Le cas d). L'associativité (10) est exprimée par

$$
\begin{aligned}
& x y z\left\{H_{0}+\sum_{i=1}^{\infty} H_{i}(y, z)\right\}\left[H_{0}+\sum_{i=1}^{\infty} H_{i}\left\{x, y z\left(H_{0}+\sum_{j=1}^{\infty} H_{j}(y, z)\right)\right\}\right] \\
\equiv & x y z\left\{H_{0}+\sum_{i=1}^{\infty} H_{i}(x, y)\right\}\left[H_{0}+\sum_{i=1}^{\infty} H_{i}\left\{x y\left(H_{0}+\sum_{j=1}^{\infty} H_{j}(x, y)\right), z\right\}\right] .
\end{aligned}
$$

Nous distinguons deux cas suivant que: i) $H_{0}=0$ ou ii) $H_{0} \neq 0$.

i) $H_{0}=0$. Supposons que

$$
H_{1} \equiv H_{2} \equiv \cdots H_{n-1} \equiv 0 \text { et } H_{n} \nRightarrow 0, \quad(n \geqq 1) .
$$

La relation $(\mathrm{F})$ devient 


$$
\begin{aligned}
& \sum_{i=n}^{\infty} H_{i}(y, z) \sum_{i=n}^{\infty} H_{i}\left\{x, y z \sum_{j=n}^{\infty} H_{j}(y, z)\right\} \\
\equiv & \sum_{i=n}^{\infty} H_{i}(x, y) \sum_{i=n}^{\infty} H_{i}\left\{x y \sum_{j=n}^{\infty} H_{j}(x, y), z\right\} .
\end{aligned}
$$

En y posant $z=0$, on a

$$
\begin{aligned}
& \sum_{i=n}^{\infty} H_{i}(y, 0) \sum_{i=n}^{\infty} H_{i}(x, 0) \\
\equiv & \sum_{i=n}^{\infty} H_{i}(x, y) \sum_{i=n}^{\infty} H_{i}\left\{x y \sum_{j=n}^{\infty} H_{j}(x, y), 0\right\},
\end{aligned}
$$

et en plus, en tenant compte du développement

$$
\sum_{i=n}^{\infty} H_{i}(x, 0) \equiv \sum_{j=m}^{\infty} h_{j} x^{j}, \quad(m \geqq n),
$$

on a

$$
\sum_{i=m}^{\infty} h_{i} x^{i} \sum_{j=m}^{\infty} h_{j} y^{j} \equiv \sum_{i=n}^{\infty} H_{i}(x, y) \sum_{j=m}^{\infty} h_{j} x^{j} y^{j}\left\{\sum_{k=n}^{\infty} H_{k}(x, y)\right\}^{j} .
$$

Le terme du moindre degré dans le premier membre de cette équation est $2 m$, tandis que celui du deuxième membre est $n+2 m+n m>2 m$. Il faut donc que le terme-là s'evanouisse et on a nécessairement

$$
\sum_{i=n}^{\infty} H_{i}(x, 0) \equiv \sum_{j=m}^{\infty} h_{j} x^{j} \equiv 0 .
$$

On a de même,

$$
\sum_{i=n}^{\infty} H_{i}(0, y) \equiv 0 .
$$

Ces deux relations impliquent que $f(x, y)$ s'écrit sous la forme

$$
f(x, y) \equiv x^{2} y^{2} \sum_{i=0}^{\infty} G_{i}(x, y),
$$

$G_{i}(x, y)$ étant un polynome homogène du degré $i$.

Si l'on continue successivement ce procédé, on obtient enfin $f(x, y)$ $=0 \mathrm{ou}$

$$
f(x, y) \equiv x^{n} y^{n} \sum_{i=0}^{\infty} K_{i}(x, y), \quad(n \geqq 2),
$$

où $K_{i}$ désigne un polynome homogène du degré $i$ avec $K_{0} \neq 0$. 
La relation $(\mathrm{F})$ sera réduit alors à

$$
\begin{aligned}
& x^{n} y^{n 2} z^{n 2}\left\{K_{0}+\cdots\right\}^{n}\left[K_{0}+\cdots\right] \\
& \quad=x^{n 2} y^{n 2} z^{n}\left\{K_{0}+\cdots\right\}^{n}\left[K_{0}+\cdots\right]
\end{aligned}
$$

où $\cdots$ ne contient que des termes du degré $\geqq 1$. En comparant les premiers termes des deux membres, on obtiendrait, si $K_{0} \neq 0$,

$$
K_{0}^{n+1} x^{n} y^{2} z^{n 2}=K_{0}^{n+1} x^{n 2} y^{n 2} z^{n},
$$

ce qui exigerait $n^{2}=n$, contrairement à $n \geqq 2$. On a donc $H_{0}=0$, ce qui entraîne le cas trivial: $f(x, y) \equiv 0$.

ii) $H_{0} \neq 0$. Supposons encore que

$$
H_{1} \equiv H_{2} \equiv \cdots \equiv H_{n-1}=0 \text { et } H_{n} \neq 0, \quad(n \geqq 1) .
$$

L'équation (F) se développe comme suit

$$
\begin{aligned}
& H_{0} \sum_{i=n}^{\infty} H_{i}(y, z)+H_{0} \sum_{i=n}^{\infty} H_{i}\left\{x, y z \sum_{j=0}^{\infty} H_{j}(y, z)\right\} \\
& \quad+\sum_{i=n}^{\infty} H_{i}(y, z) \sum_{i=n}^{\infty} H_{i}\left\{x, y z \sum_{j=0}^{\infty} H_{j}(y, z)\right\} \\
& \equiv H_{0} \sum_{i=n}^{\infty} H_{i}(x, y)+H_{0} \sum_{i=n}^{\infty} H_{i}\left\{x y \sum_{j=0}^{\infty} H_{j}(x, y), z\right\} \\
& \quad+\sum_{i=n}^{\infty} H_{i}(x, y) \sum_{i=n}^{\infty} H_{i}\left\{x y \sum_{j=0}^{\infty} H_{j}(x, y), z\right\} .
\end{aligned}
$$

Si l'on divise cette équation, par $H_{0}$ et y pose $z=x$, on aura

$$
\begin{aligned}
\sum_{i=n}^{\infty} H_{i}(x, y)-\sum_{i=n}^{\infty} H_{i}(y, x) \\
=\sum_{i=n}^{\infty} H_{i}\left\{x, y x \sum_{j=0}^{\infty} H_{j}(y, x)\right\}-\sum_{i=n}^{\infty} H_{i}\left\{x y \sum_{j=0}^{\infty} H_{j}(x, y), x\right\} \\
\quad+\frac{1}{H_{0}} \sum_{i=n}^{\infty} H_{i}(y, x) \sum_{i=n}^{\infty} H_{i}\left\{x, y x \sum_{j=0}^{\infty} H_{j}(y, x)\right\} \\
\quad-\frac{1}{H_{0}} \sum_{i=n}^{\infty} H_{i}(x, y) \sum_{i=0}^{\infty} H_{i}\left\{x y \sum_{j=0}^{\infty} H_{j}(x, y), x\right\} .
\end{aligned}
$$

Alors, on se sert des polynomes nouveaux suivants $J_{i}$ et $G_{i}$ avec $i$ comme degré commun

$$
H_{i}(x, y) \equiv a_{i} x^{i}+b_{i} y^{i}+J_{i}(x, y)
$$

et 


$$
G_{i}(x, y) \equiv J_{i}(x, y)-J_{i}(y, x)
$$

où

$$
J_{i}(0, y) \equiv J_{i}(x, 0) \equiv 0 \quad \text { et } \quad i=n, n+1, \cdots .
$$

Nous considérons maintenant les termes du degré $n$ du (G)

$$
a_{n} x^{n}+b_{n} y^{n}-a_{n} y^{n}-b_{n} x^{n}+G_{n}(x, y) \equiv a_{n} x^{n}-b_{n} x^{n} .
$$

Nous avons l'égalité

$$
\left(b_{n}-a_{n}\right) y^{n}+G_{n}(x, y) \equiv 0 .
$$

Ceci entraîne l'égalité:

$$
a_{n}=b_{n} \text { et } G_{n}(x, y) \equiv 0 .
$$

D’une manière générale, si les deux églités suivantes sont vraies

$$
a_{i}=b_{i} \quad \text { et } \quad G_{i}(x, y) \equiv 0
$$

pour $n \leqq i \leqq m$, les termes du degré $m+1$ dans (G) seront

$$
\begin{aligned}
& a_{m+1} x^{m+1}+b_{m+1} y^{m+1}-a_{m+1} y^{m+1}-b_{m+1} x^{m+1}+G_{m+1}(x, y) \\
& \quad=a_{m+1} x^{m+1}-b_{m+1} x^{i n+1},
\end{aligned}
$$

car les autres termes du degré $m+1$ s'évanouissent en vertu de la symétrie de $H_{i}(x, y)$ et de $H_{j}(x, y)$ pour $i, j \leqq m$.

Par conséquent, nous avons seulement

$$
\left(b_{m+1}-a_{m+1}\right) y^{m+1}+G_{m+1}(x, y) \equiv 0,
$$

ce qui entraîne

$$
a_{m+1}=b_{m+1} \quad \text { et } \quad G_{m+1}(x, y) \equiv 0 .
$$

C'est-à-dire, on a vérifié que $H_{i}(x, y)$ est́ symétrique par rapport à $x$ et $y$ pour tous les entiers $i(\geqq n)$, ou, autrement dit,

$$
\sum_{i=0}^{\infty} H_{i}(x, y)=\sum_{i=0}^{\infty} H_{i}(y, x) \text {. }
$$

Il s'ensuit que d) du théorème 6 , où $S_{2}(x, y)$ est ou bien symétrique et s'annule en $(0,0)$, ou bien $S_{2}(x, y)=0$.

C.Q.F.D.

\subsection{Exemples et remarques.}

D'abord il y a en effet la fonction de la forme 3) ou 4) du théorème 6 . 


\section{Exemple de 3).}

$$
f(x, y)=\frac{x+y}{1-x y}=(x+y)(1+x y+x y+\cdots) .
$$

\section{Exemple de 4).}

$$
f(x, y)=\frac{x y}{1+x+y}=x y\left\{1-(x+y)+(x+y)^{2}-\cdots\right\} .
$$

Puis, dans le cas 3 ), on peut exprimer la fonction $f\left(x, y^{\prime}\right)$ par la forme

$$
f(x, y) \equiv \varphi^{-1}\{\varphi(x)+\varphi(y)\}
$$

dans un voisinage de $(0,0)$, d'après la théorie du groupe de Lie, où $\varphi(x)$ désigne une fonction continue, parce qu'on peut établir qu'elle est symétrique dans un voisinage d'un point, et on peut vérifier qu'əlie est symétrique dans tout le domaine de la fonction analytique par le prolongement analytique.

\section{§5. Sollutions des équations dur ramg un par l’intégration.}

\subsection{Une équation fonctionnelle du rang un. [10]}

Comıne un exemple d'équation du rang un, nous considérons l'équation fonctionnelle suivante, qui n'est pas dérivée directement de l'équation de Cauchy.

$$
f(x)=\frac{1}{n}\left\{f\left(\frac{x}{n}\right)+f\left(\frac{x+1}{n}\right)+\cdots+f\left(\frac{x+n-1}{n}\right)\right\},
$$

C'est du type $(1, n, 1)$. Appliquant la relation (26) à chaque terme du second membre de la même relation, nous obtenons une équation qui est semblable à (26) et du type $\left(1, n^{2}, 1\right)$ :

$$
f(x)=\frac{1}{n^{2}}\left\{f\left(\frac{x}{n^{2}}\right)+f\left(\frac{x+1}{n^{2}}\right)+\cdots+f\left(\frac{x+n^{2}-1}{n^{2}}\right)\right\} .
$$

Après avoir répété $m$ fois ce procédé, nous arrivons à la relation

$$
f(x)=\frac{1}{n^{m}}\left\{f\left(\frac{x}{n^{m}}\right)+f\left(\frac{x+1}{n^{m}}\right)+\cdots+f\left(\frac{x+n^{m}-1}{n^{m}}\right)\right\} .
$$


Si la fonction $f(x)$ est continue (ou sommable) sur l'intervalle fermé $[0,1]$, nous aurons, à la limite de $m \rightarrow \infty$, une intégrale définie dedans le second membre et en même temps la solution.

$$
f(x)=\int_{0}^{1} f(x) d x=c, \quad(c: \text { constante arbitraire }) .
$$

Ceci remplit sûrement l'équation (26).

L'équation dont le coefficient du second membre est égal à un est

$$
f(x)=f\left(\frac{x}{n}\right)+f\left(\frac{x+1}{n}\right)+\cdots+f\left(\frac{x+n-1}{n}\right) .
$$

La différentiation par rapport à $x$, si ce soit possible, nous amène à la même équation que (26)

$$
f^{\prime}(x)=\frac{1}{n}\left\{f^{\prime}\left(\frac{x}{n}\right)+f^{\prime}\left(\frac{x+1}{n}\right)+\cdots+f^{\prime}\left(\frac{x+n-1}{n}\right)\right\} .
$$

Comme la solution est $f^{\prime}(x)=c_{1}, f(x)$ est donné par

$$
f(x)=c_{1} x+c_{0}, \quad\left(c_{0}, c_{1}: \text { constantes arbitraires }\right) .
$$

En portant cette expression dans l'équation (27), nous trouvons $c_{1}+2 c_{0}$ $=0$. On a donc

$$
f(x)=c\left(x-\frac{1}{2}\right), \quad(c \text { : constante arbitraire }) .
$$

\subsection{Solutions de l'équation d'une variable.}

Dans le cas général d'une variable, nous avons obtenu le résultat ci-dessous dont la vérification nous renverrons à notre article original [10].

Théorème 8. Les solution de l'équation fonctionnelle

$$
f(x)=k \sum_{i=1}^{n} f\left\{\frac{x+(n-i) a+(i-1) b}{n}\right\}
$$

où la fonction inconnue $f(x)$ appartient à la classe $C^{p}$ sur $[a, b]$ et où le nombre naturel $p$ satisfait aux inégalités:

$$
n^{p-2}<k \leqq n^{p-1}
$$

sont données comme suit. 
Sur les équations fonctionnelles généralisées de Cauchy

1) Cas où $k$ est égal à $n^{p-1}$. Elles sont les polynomes du degré $p$

$$
f(x)=c\left(x^{p}+c_{1} x^{p-1}+\cdots+c_{p}\right)
$$

où $c$ est une constante arbitraire et $c_{1}, c_{2}, \cdots, c_{p}$ les constantes déterminées par le système des $p$ équations:

$$
\int_{a}^{b} f^{(q)}(x) d x=0, \quad(q=0,1,2, \cdots, p-1) .
$$

2) Cas où $k$ n'est pas égal à $n^{p-1}$. Elles sont triziales, c'est-à-dire, elles sont identiquement nulles.

\section{3. Solutions de l'équation de deux variables. [11]}

Nous étendrons le théorème 8 au cas d'une fonction inconnue de deux variables, mais l'extension au cas de $n(\geqq 3)$ variables est possible sans aucune modification essentielle dans les raisonnements.

Théorème 9. L'équation fonctionnelle du type (2, mn, 1):

$$
\begin{aligned}
f(x, y)=k \sum_{i=1}^{m} \sum_{j=1}^{n} f\left\{\frac{x+(m-i) a+(i-1) b}{m},\right. \\
\left.\frac{y+(n-j) c+(j-1) d}{n}\right\},
\end{aligned}
$$

où la fonction inconnue $f(x, y)$ admet les dérivées partielles continues jusqu'à l'ordre suffisamment haut dans le rectangle $I:[a, b] \times[c, d]$, admet une solution non triviale qui est un polynome de degré $p$ en $x$ et $q$ en $y$, avec un facteur constant et arbitraire, dans le cas seulement où le paramètre $k$ est égal à $m^{p-1} n^{q-1}$, p et q étant deux nombres naturels ou nuls.

Dans le cas de deux variables, il y aura les conditions

$$
\iint_{I} \frac{\partial^{r+s} f(x, y)}{\partial x^{r} \partial y^{s}} d x d y=0, \quad\left(m^{r} n^{s}<m^{p} n^{q}\right)
$$

pour déterminer tous les coefficients de la solution de (29).

Enfin nous remarquons que, employant les solutions $f_{r}(x)$ de (28) pour $k=n^{r-1}$, nous pourrons représenter les solutions $f_{p, q}(x, y)$ de (29), pour chaque paire $(p, q)$ telle que $k=m^{p-1} n^{q-1}$, sous la forme 


$$
f_{p, q}(x, y)=f_{p}(x) f_{q}(y)
$$

puisque le produit du second membre remplit effectivement la condition (I).

\section{BIBLHOGRAPHIE}

[1] J. Aczél, Sur les opérations définies pour nombres réels, Bull. Soc. Math. France, 76 (1949), 59-64.

[2] J. Aczél, Vorlesungen über Funktionalgleichungen und ihre Anwendungen, Birkhäuser Verlag, Bazel und Stuttgart, (1961).

[3] W. Eichhorn, Über die multiplikative Cauchysche Funktionalgleichung für die Hamiltonsche Quaternionen und die Cayleyschen Zahles, J. für die reine u. angewandte Math, 221 (1966), 2-13.

[4] M. Kuczma, A survey of the theory of the functional equations, Univ. Beograd, Publ. Electrotehn. Fak. Ser. Mat. i Fiz. 130 (1964), 1-64.

[5] A. Kuwagaki, Sur l'équation fonctinnelle: $f(x+y)=R\{f(x), f(y)\}$, Mem. Coll. Sci. Univ. Kyoto, 26 (1951), 139-144.

[6] A. Kuwagaki, Sur l'équation fonctionnelle de Cauchy pour les matrices, J. Math. Soc. Japan, $\mathbb{1 4}$ (1962), 359-366.

[7] A. Kuwagaki, Sur l'équation fonctionnelle rationnelle de la fonction inconnue de deux variables, Mem. Coll. Sci. Univ. Kyoto, 27 (1952), 145-151.

[8] A. Kuwagaki, Sur les fonctions de deux variables satisfaisant une formule d'addition algébrique, Mem. Coll. Sci. Univ. Kyoto, 27 (1952), 139-143.

[9] A. Kuwagaki, Sur la fonction analytique de deux variables complexes satisfaisant l'associativité: $f\{x, f(y, z)\}=f\{f(x, y), z\}$, Mem. Coll. Sci. Univ. Kyoto, 27 (1953), 225-234.

[10] A. Kuwagaki, Sur quelques équations fonctionnelles et leurs solutions caractéristiques I, Mem. Coll. Sci. Univ. Kyoto, 26 (1951), 271-277.

[11] A. Kuwagaki, Sur quelques équations fonctionnelles et leurs solutions caractéristiques II, Mem. Coll. Sci. Univ. Kyoto, 27 (1952), 47-53.

[12] O. Perron, Über eine für die Invariantentheorie wichtige Funktionalgleichung, Math. Z., 48 (1942), 136-172. 